\title{
Evaluation of the Etest and disk diffusion method for detection of the activity of ceftazidime-avibactam against Enterobacterales and Pseudomonas aeruginosa in China
}

Qi Wang, Feifei Zhang, Zhanwei Wang, Hongbin Chen, Xiaojuan Wang, Yawei Zhang, Shuguang Li and Hui Wang ${ }^{*}$ (D)

\begin{abstract}
Background: Ceftazidime-avibactam was approved in China in 2019 for treating complicated intra-abdominal infections, hospital-acquired pneumonia, ventilator-associated pneumonia, and infections caused by Enterobacterales and Pseudomonas aeruginosa for which treatment options are limited. However, no currently available commercial systems have been approved for antimicrobial susceptibility testing of ceftazidime-avibactam in China. Here, we evaluated the Etest and disk diffusion method for detecting the activity of ceftazidime-avibactam against Enterobacterales and P. aeruginosa in China.

Results: In total, 194 Enterobacterales and 77 P. aeruginosa isolates, which were divided into a random selection group (140 Enterobacterales and 46 P. aeruginosa isolates) and stock group (54 Enterobacterales and 31 P. aeruginosa isolates), were assessed by the Etest, disk diffusion and broth microdilution methods. Minimum inhibitory concentrations and zone diameters were interpreted according to the CLSI supplement M100 30th edition. For all 271 tested isolates, no very major errors were found by using Etest, whereas the overall major error rate was 2.0\% (4/203). The overall categorical agreement rates of Etest for Enterobacterales and P. aeruginosa were 99.5\% (193/194) and $96.1 \%$ (74/77), respectively, and the essential agreement rates were $95.9 \%(186 / 194)$ and $94.8 \%$ (73/77), respectively. The disk diffusion method showed that the very major error and major error rates were 1.5\% (3/204) and 2.5\% (5/203), respectively. Overall categorical agreement rates values of the disk diffusion method for Enterobacterales and P. aeruginosa were 98.5\% (191/194) and 93.5\% (72/77) compared with broth microdilution, respectively.

Conclusions: For Enterobacterales and P. aeruginosa, both the Etest and disk diffusion method showed acceptable performance as alternatives to the standard broth microdilution method for clinical treatment interpretation. Application of the disk diffusion method in Enterobacterales was slightly better than that in P. aeruginosa.
\end{abstract}

Keywords: Ceftazidime-avibactam, Etest, Broth microdilution, Disk diffusion

\footnotetext{
* Correspondence: whuibj@163.com

Department of Clinical Laboratory, Peking University People's Hospital, Beijing 100044, China
}

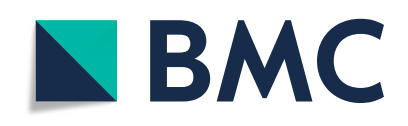

(c) The Author(s). 2020 Open Access This article is licensed under a Creative Commons Attribution 4.0 International License, which permits use, sharing, adaptation, distribution and reproduction in any medium or format, as long as you give appropriate credit to the original author(s) and the source, provide a link to the Creative Commons licence, and indicate if changes were made. The images or other third party material in this article are included in the article's Creative Commons licence, unless indicated otherwise in a credit line to the material. If material is not included in the article's Creative Commons licence and your intended use is not permitted by statutory regulation or exceeds the permitted use, you will need to obtain permission directly from the copyright holder. To view a copy of this licence, visit http://creativecommons.org/licenses/by/4.0/ The Creative Commons Public Domain Dedication waiver (http://creativecommons.org/publicdomain/zero/1.0/) applies to the data made available in this article, unless otherwise stated in a credit line to the data. 


\section{Background}

Gram-negative bacilli, particularly carbapenem-resistant Enterobacterales (CRE) and Pseudomonas aeruginosa, exhibit major antimicrobial resistance worldwide, including in European countries, the United States (USA), and China [1-3]. The approval of ceftazidime-avibactam for clinical use in Europe and the USA has provided new treatment options for CRE-infected patients, particularly those with serine-carbapenemase resistance mechanisms $[4,5]$. Ceftazidime-avibactam was approved in China in 2019 for treating complicated intra-abdominal infections, hospital-acquired pneumonia, ventilator-associated pneumonia, and infections caused by Enterobacterales and $P$. aeruginosa without enough effective treatment options. In China, there is currently no automated system for antimicrobial susceptibility testing of ceftazidime-avibactam. Ceftazidime-avibactam susceptibility tests are urgently needed to facilitate appropriate targeted treatment in patients with infections caused by multidrug-resistant Enterobacterales and P. aeruginosa. Although ceftazidime-avibactam has excellent in vitro activity against carbapenem-resistant Klebsiella pneumoniae (CRKP) and $P$. aeruginosa isolates, a few resistant isolates remain during treatment or nosocomial infection transmission [6-8]. Therefore, the susceptibility test results for ceftazidime-avibactam are even more critical.

In most laboratories in China, performing the standard broth microdilution (BMD) method is challenging. Therefore, alternative methods are needed to reliably determine ceftazidime-avibactam susceptibility. In this study, we evaluated two antimicrobial susceptibility testing methods for ceftazidime-avibactam, i.e., the Etest and disk diffusion method with the reference BMD, to evaluate whether these simple methods can be utilized for testing ceftazidime-avibactam in the clinical setting.

\section{Results}

Etest versus the BMD

According to the BMD method, 24.2\% (47/194) of Enterobacterales and $28.4 \%(21 / 74)$ of $P$. aeruginosa were resistant to ceftazidime-avibactam. For all 194 Enterobacterales isolates, no very major error (VME) were found using the Etest. One isolate in the stock group was classified as resistant by Etest but susceptible by BMD. The major error (ME) rate was $0.7 \%(1 / 147)$ in Enterobacterales. As shown in Table 1, the overall categorical agreement (CA) rate was $99.5 \%$, and the overall essential agreement (EA) rate was $95.9 \%$. The CA rate of the stock group was $98.1 \%$, and that in the random selection group was $97.9 \%$. When comparing Etest results with the BMD results, the MICs of nine isolates exceeded the two-fold dilution. As shown in Fig. 1, the Etest minimum inhibitory concentrations (MICs) of 102 (52.5\%) isolates were consistent with the BMD MICs. The Etest MICs of 71 (36.6\%) isolates were one-fold dilution higher than the BMD MICs. Only 12 (6.2\%) isolates showed Etest MICs that were one-fold dilution lower than the BMD MICs.

For $77 P$. aeruginosa isolates, no VME were found using the Etest method. Three isolates in the stock group were classified as resistant by Etest but susceptible by BMD. As shown in Table 1, the overall CA and EA rates were $96.1 \%$ and $94.8 \%$, respectively. The CA rates in the stock and random selection groups were $90.3 \%$ and $100 \%$, respectively. As shown in Fig. 2, the Etest MICs of 45 (58.4\%) isolates were consistent with those obtained by the BMD. The Etest MICs of 22 (28.6\%) isolates were one-fold dilution higher than those obtained by the BMD, whereas those of six $(7.8 \%)$ isolates were one-fold dilution lower than the MICs obtained by the BMD. For one strain, the Etest MIC was two-fold

Table 1 Evaluation of essential and categorical agreement between the BMD method and Etest or disk diffusion method for analysis of ceftazidime-avibactam antimicrobial susceptibility

\begin{tabular}{|c|c|c|c|c|c|c|c|c|c|}
\hline \multirow[t]{2}{*}{ Organism } & \multirow{2}{*}{$\begin{array}{l}\text { No. of } \\
\text { isolates } \\
\text { tested }\end{array}$} & \multirow{2}{*}{$\begin{array}{l}\text { No. of } \\
\text { resistant } \\
\text { isolates } \\
\text { by BMD }\end{array}$} & \multicolumn{4}{|l|}{ E-test } & \multicolumn{3}{|c|}{ Disk diffusion } \\
\hline & & & $\begin{array}{l}\text { No. }(\%) \text { of } \\
\text { CA }\end{array}$ & $\begin{array}{l}\text { No. (\%) of } \\
\text { EA }\end{array}$ & $\begin{array}{l}\text { No. (\%) of } \\
\text { VME }\end{array}$ & $\begin{array}{l}\text { No. (\%) of } \\
\text { ME }\end{array}$ & $\begin{array}{l}\text { No. (\%) of } \\
\text { CA }\end{array}$ & $\begin{array}{l}\text { No. }(\%) \text { of } \\
\text { VME }\end{array}$ & $\begin{array}{l}\text { No. (\%) of } \\
\text { ME }\end{array}$ \\
\hline \multicolumn{10}{|l|}{ Enterobacterales } \\
\hline Random selection group & 140 & 7 & $140(100)$ & $137(97.9)$ & $0(0)$ & $0(0)$ & $140(100)$ & $0(0)$ & $0(0)$ \\
\hline Stock group & 54 & 40 & $53(98.1)$ & $49(90.7)$ & $0(0)$ & $1(7.1)$ & $51(94.4)$ & $2(5.0)$ & $1(7.1)$ \\
\hline Total in Enterobacterales & 194 & 47 & $193(99.5)$ & $186(95.9)$ & $0(0)$ & $1(0.7)$ & $191(98.5)$ & $2(4.3)$ & $1(0.7)$ \\
\hline \multicolumn{10}{|l|}{$P$. aeruginosa } \\
\hline Random selection group & 46 & 6 & $46(100)$ & $45(97.8)$ & $0(0)$ & $0(0)$ & $43(93.5)$ & $0(0)$ & $3(7.5)$ \\
\hline Stock group & 31 & 15 & $28(90.3)$ & $28(90.3)$ & $0(0)$ & $3(18.7)$ & $29(93.5)$ & $1(6.7)$ & $1(6.3)$ \\
\hline Total in $P$. aeruginosa & 77 & 21 & $74(96.1)$ & $73(94.8)$ & $0(0)$ & $3(5.4)$ & $72(93.5)$ & $1(4.8)$ & $4(7.1)$ \\
\hline $\begin{array}{l}\text { Total in all tested } \\
\text { isolates }\end{array}$ & 271 & 68 & $267(98.5)$ & $259(95.6)$ & $0(0)$ & $4(2.0)$ & $263(97.0)$ & $3(1.5)$ & $5(2.5)$ \\
\hline
\end{tabular}

EA essential agreement, CA categorical agreement, VME very major error (false susceptible), ME major error (false resistant) 


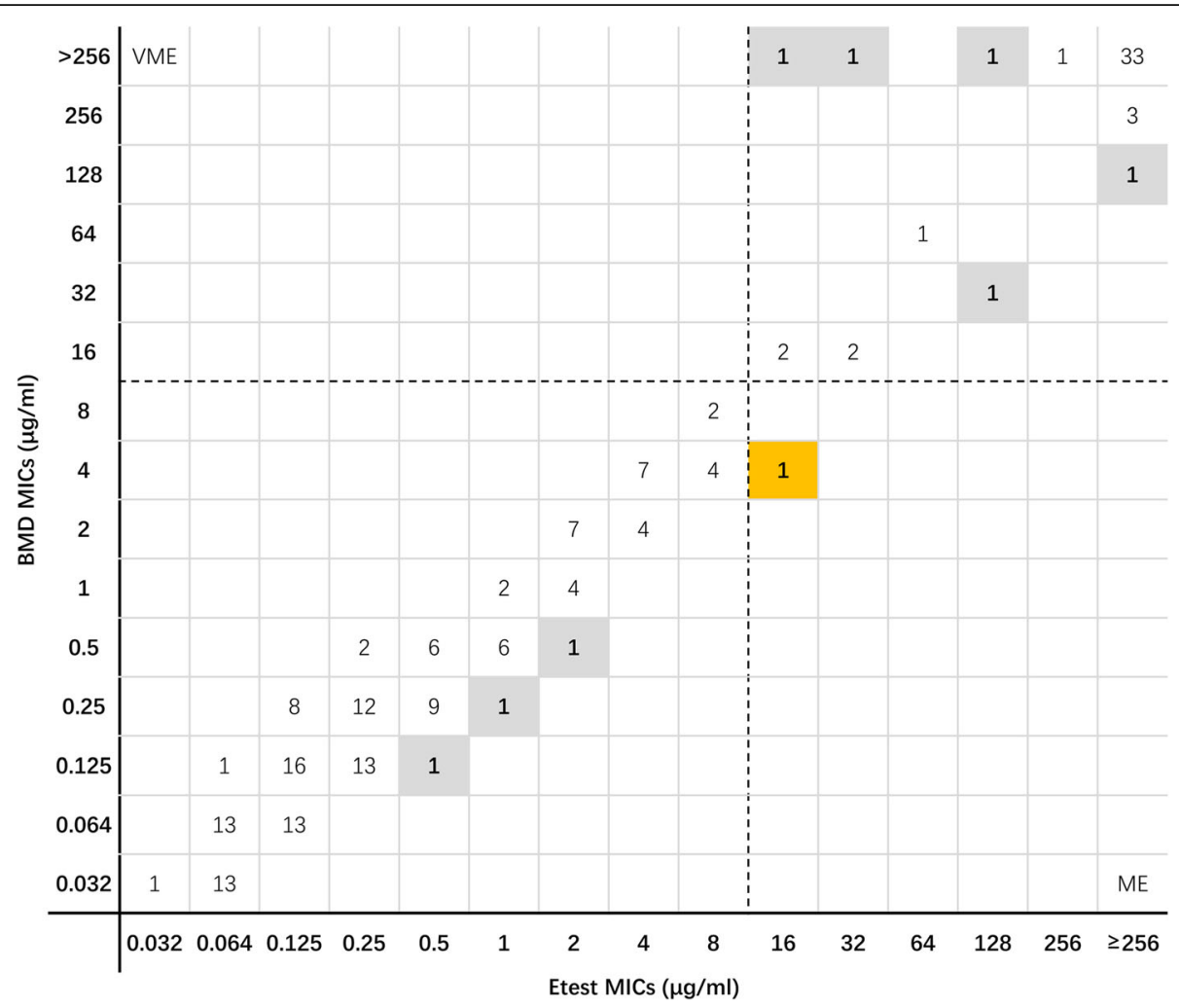

Fig. 1 Scatter plot of ceftazidime-avibactam Etest MICs versus BMD MICs against Enterobacterales. Dotted lines represent the susceptibility breakpoint for ceftazidime-avibactam. VME: very major error (false susceptible); ME: major error (false resistant). The gray background indicates that the MIC of the Etest did not satisfy the essential agreement compared with the MIC of the BMD; the yellow background indicates that a major error occurred in the MIC of the Etest compared with the MIC of the BMD

dilutions higher than that obtained by the BMD. Three MEs appeared in the stock group when the Etest method was used.

\section{Disk diffusion method versus BMD}

A comparison of the disk diffusion method and BMD results for 194 Enterobacterales isolates are shown in Fig. 3. The overall CA rate in the 194 Enterobacterales isolates was $98.5 \%$. Two VMEs and $1 \mathrm{ME}$ were found using the disk diffusion method in the stock group; all were carbapenem-resistant $K$. pneumoniae carbapenemase (KPC)-producing isolates. No VME or ME were found using the disk diffusion method in the random selection group. There were 22 isolates of Enterobacterales with zone diameters between 19 and $22 \mathrm{~mm}$. Forty-one of the 47 resistant isolates obtained by BMD showed zone diameters in the range of $13-20 \mathrm{~mm}$.

Comparison of the disk diffusion method and BMD for $77 P$. aeruginosa isolates showed that the ME\% was $7.1 \%$. As shown in Table 1, the overall CA rate was 93.5\%. Moreover, as shown in Fig. 4, when the zone diameter was $20 \mathrm{~mm}$, the MICs obtained by the BMD method were $4,8,16,32$, and $64 \mu \mathrm{g} / \mathrm{mL}$.

\section{Discussion}

In the past decade, the incidence of CRE, particularly CRKP, has increased significantly in China. The latest China Antimicrobial Resistance Surveillance System data showed that the incidence of CRKP nationwide was as high as $10.1 \%$ (http://www.carss.cn/Report/Details?aId= 648). However, few active antibacterial agents, such as tigecycline and colistin, are available to treat CRKP in the clinical setting, resulting in high mortality worldwide $[9,10]$. Previous molecular epidemiological data revealed that more than 70\% of CRE isolated in China from 2012 to 2016 produce KPC-type carbapenemases [11]. Ceftazidime-avibactam, a drug with potent antibacterial activity against serine-carbapenemase, was approved for use in China in 2019. Recent retrospective studies showed that ceftazidime-avibactam treatment of CRKP and $P$. aeruginosa infection in patients who have undergone solid organ transplantation improves clinical success rates $[12,13]$. Despite these promising findings, ceftazidime-avibactam antimicrobial susceptibility test results are essential for the clinical use of this treatment. Additionally, commercial automated systems in the clinical microbiology laboratory cannot be used for 


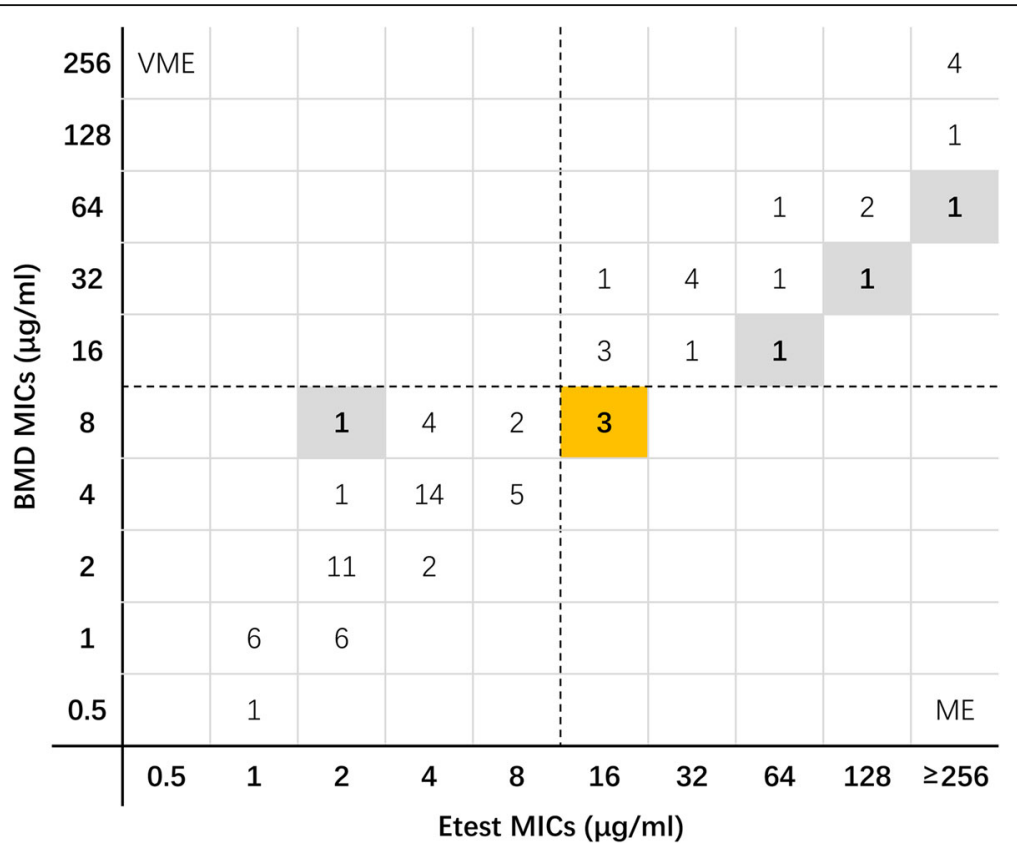

Fig. 2 Scatter plot of ceftazidime-avibactam Etest MICs versus BMD MICs against Pseudomonas aeruginosa. Dotted lines represent the susceptibility breakpoint for ceftazidime-avibactam. VME: very major error (false susceptible); ME: major error (false resistant). The gray background indicates that the MIC of the Etest did not satisfy the essential agreement compared with the MIC of the BMD; the yellow background indicates that three major errors occurred in the MIC of the Etest compared with the MIC of the BMD

antimicrobial susceptibility testing of ceftazidimeavibactam in China.

This is the first study to compare the Etest, disk diffusion method, and BMD to detect ceftazidime-avibactam susceptibility in China. We use BMD as a standard clinical method for comparison with the other methods. Compared with the standard BMD method, no VME were found using the Etest method. The results of Etest MICs and BMD MICs were reasonably well correlated for both Enterobacterales and P. aeruginosa. The overall

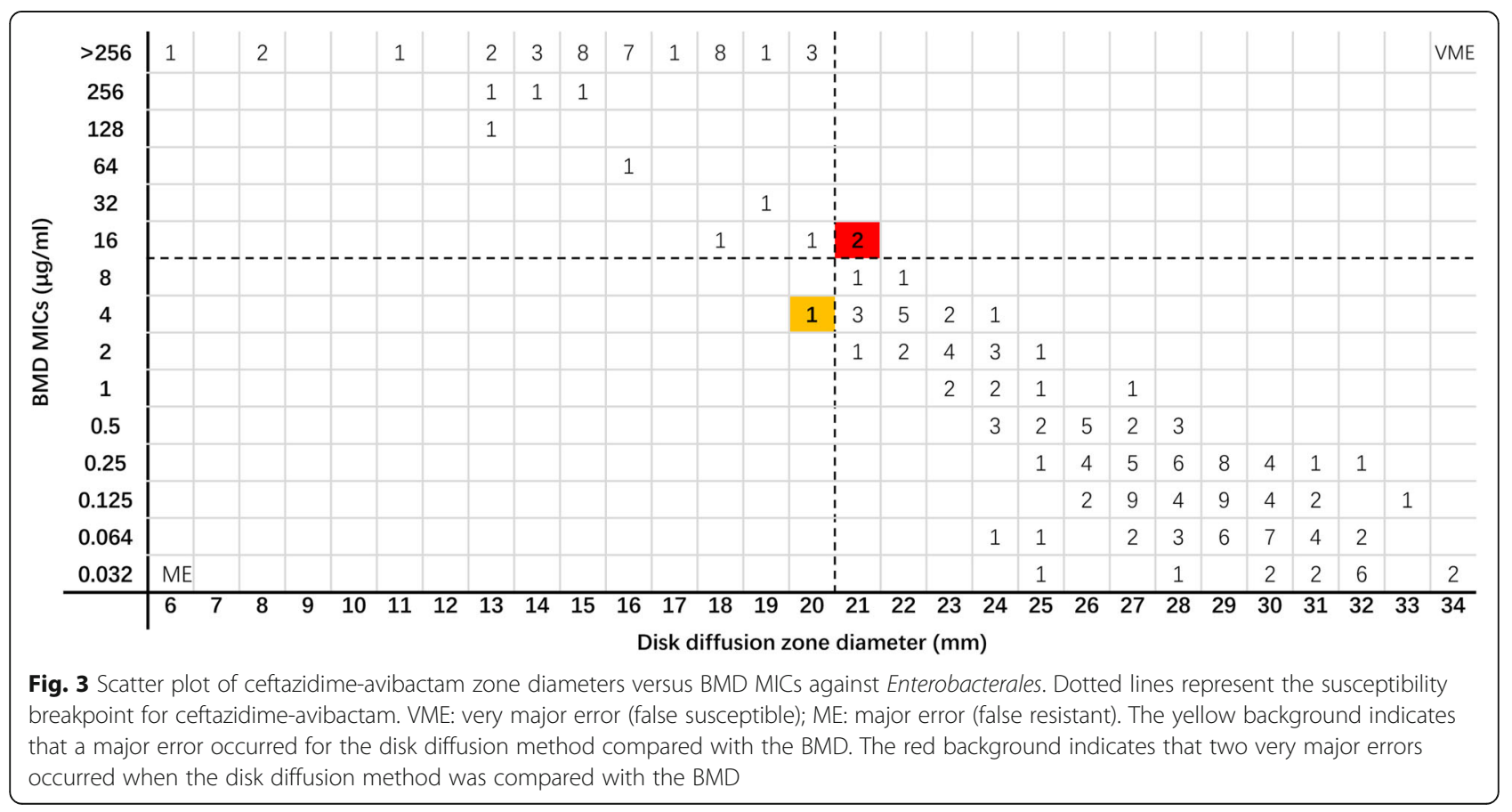




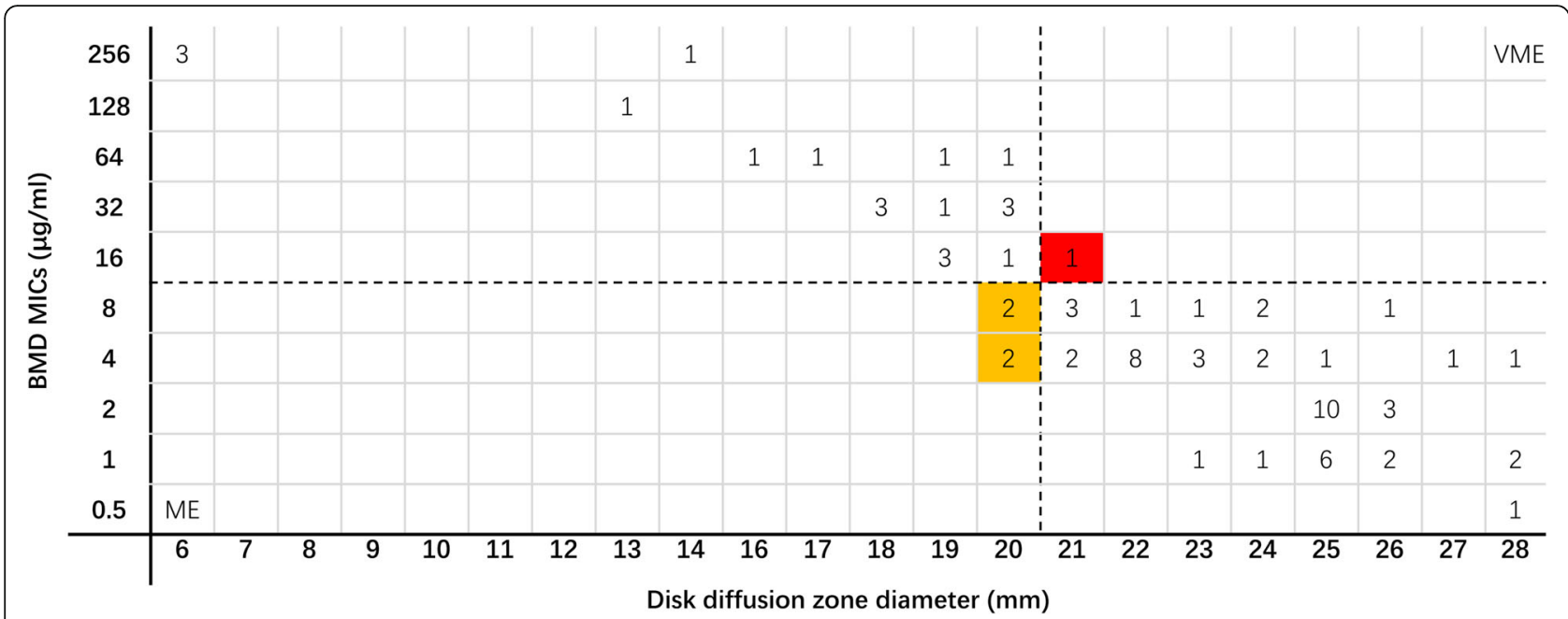

Fig. 4 Scatter plot of ceftazidime-avibactam zone diameters versus BMD MICs against Pseudomonas aeruginosa. Dotted lines represent the susceptibility breakpoint for ceftazidime-avibactam. VME: very major error (false susceptible); ME: major error (false resistant). The yellow background indicates that four major errors occurred for the disk diffusion method compared with the BMD. The red background indicates that a very major error occurred when the disk diffusion method was compared with the BMD

EA\% of 271 tested isolates was 95.6\%. Compared with the BMD method, the Etest method exhibited an excellent linear correlation, supporting the use of this approach as an alternative to the standard clinical method without considering economic costs. The Etest results were similar to those of previous research findings [14]. A study of the ceftazidime-avibactam Etest in Germany reported excellent results in terms of EA and CA for susceptibility testing of Enterobacterales and $P$. aeruginosa [15]. A total of 140 Enterobacterales and 60 P. aeruginosa were examined; the total EA\% and CA\% were $99.0 \%$ and $99.5 \%$, respectively.

The disk diffusion method is easy to implement in the clinical setting from an economic perspective. The CA\% values of the ceftazidime-avibactam disk diffusion method against Enterobacterales and $P$. aeruginosa were $98.5 \%$ and $93.5 \%$, respectively. However, a previous study by Shields et al. showed that $28 \%$ of ceftazidimeavibactam susceptible CRE isolates were classified as resistant by disk diffusion. The ME\% is significantly higher than that obtained in our study [14]. This difference in data may be related to differences in the disk manufacturer. Indeed, our results showed that application of the disk diffusion method was more appropriate for Enterobacterales than for $P$. aeruginosa. Notably, however, the disk diffusion method did not exhibit an excellent linear correlation with BMD. Moreover, for both Enterobacterales or $P$. aeruginosa against ceftazidime-avibactam, the disk diffusion method tends to show falsely susceptible results. As the sample size in our study was small, the number of VME was low. Therefore, clinical verification of larger sample sizes is needed.
Based on our results, when the zone diameters of ceftazidime-avibactam against Enterobacterales and $P$. aeruginosa were $20-21 \mathrm{~mm}$, BMD testing should be performed to avoid false-susceptible or false-resistant results. Our results were consistent with those of other studies [16, 17]. This should also be considered when users refer to the new version of CLSI M100, which suggests using the disk diffusion method for Enterobacterales [18]. The CLSI recommends that MICs should be determined if the zone for Enterobacterales isolates is 20-22 mm. CLSI does not have a similar comment for P. aeruginosa.

\section{Conclusions}

In conclusion, for Enterobacterales and P. aeruginosa, ceftazidime-avibactam Etest and the disk diffusion method showed acceptable performance as alternatives to the BMD method for clinical treatment interpretation. Application of the disk diffusion method for Enterobacterales was slightly better than that for $P$. aeruginosa.

\section{Methods}

\section{Bacterial groups}

Isolates were divided into two groups, i.e., random selection group and stock group (Table S1). For the random selection group, we randomly selected 140 Enterobacterales and $46 P$. aeruginosa isolates from clinical nonrepeated isolates obtained from Peking University People's Hospital. Among these isolates, 59.3\% (83/140) of Enterobacterales and 56.5\% (26/46) of P. aeruginosa isolates were defined as fresh clinical isolates obtained within 1 month prior to testing (November 2019 to 
March 2020). Based on the collection of fresh clinical isolates, we conducted three batches of antimicrobial susceptibility tests, each in parallel with three methods (BMD, Etest, and disk diffusion method). The first test was conducted in December 2019, and the testing isolates were isolated within 1 month before the test date. The second and third tests were conducted in January 2020 and March 2020, respectively, and the test isolates were isolated within 1 month before the test date. The remaining isolates in the random selection group were obtained from the strain repository of Peking University People's Hospital from January 2018 to October 2019. The 140 isolates of Enterobacterales used for testing included 13 species, i.e., 25 K. pneumoniae, 19 Escherichia coli, 18 Proteus mirabilis, 17 Enterobacter cloacae, 16 Serratia marcescens, 15 Citrobacter freundii, $14 \mathrm{~K}$. oxytoca, 4 Proteus vulgaris, 3 Morganella morganii, 3 Providencia stuartii, 2 Providencia rettgeri, $2 K$. aerogenes, and 2 Citrobacter koseri isolates. For the stock group, we selected 54 isolates of Enterobacterales from 15 hospitals in the CRE China-Network from January 2015 to October 2019 and requested MICs of ceftazidime-avibactam of $2->256 \mu \mathrm{g} / \mathrm{mL}$. Among these isolates, six (11.1\%) showed MICs for ceftazidime-avibactam of $8-16 \mu \mathrm{g} / \mathrm{mL}$, and 15 isolates (27.8\%) showed MICs of $4-32 \mu \mathrm{g} / \mathrm{mL}$. These isolates with known MICs were mainly used to verify the accuracy of the value near the ceftazidimeavibactam breakpoint. The carbapenem-resistance genes present in these isolates were determined in previous studies [11]. The 54 isolates of Enterobacterales used in this study included $29 \mathrm{~K}$. pneumoniae (18 with bla $a_{\mathrm{KPC}}$ and 10 with bla $\left.a_{\mathrm{NDM}}\right), 12$ E. coli (2 with bla $a_{\mathrm{KPC}}$ and 7 with $\left.b l a_{\mathrm{NDM}}\right), 8$ E. cloacae (1 with $b l a_{\mathrm{KPC}}, 5$ with bla $a_{\mathrm{NDM}}, 1$ with $b l a_{\mathrm{IMP}}$, and 1 with bla $\left.a_{\mathrm{VIM}}\right), 3 \mathrm{~K}$. oxytoca (2 with $b l a_{\mathrm{IMP}}$ and 1 with $b l a_{\mathrm{NDM}}$ ), and 2 Citrobacter freundii (1 with $b l a_{\mathrm{IMP}}$ and 1 with $b l a_{\mathrm{NDM}}$ ).

We selected 31 isolates of $P$. aeruginosa from the 8 hospitals involved in the Chinese Antimicrobial Resistance Surveillance of Nosocomial infections 2018 project as stock group isolates. The MICs of ceftazidimeavibactam were $2->256 \mu \mathrm{g} / \mathrm{mL}$. Among these isolates, 12 (38.7\%) had MICs for ceftazidime-avibactam between 8 and $16 \mu \mathrm{g} / \mathrm{mL}$ and 25 (80.6\%) had MICs between 4 and $32 \mu \mathrm{g} / \mathrm{mL}$. These $P$. aeruginosa isolates with known MICs are mainly used to verify the accuracy of the value near the ceftazidime-avibactam breakpoint.

All isolates were removed from $\mathrm{a}-80^{\circ} \mathrm{C}$ ultra-low temperature freezer and transferred to Columbia blood agar twice before antimicrobial susceptibility testing.

\section{Antimicrobial susceptibility testing}

For the disk diffusion method, ceftazidime-avibactam disks were obtained from Oxoid (Hampshire, UK). The content of ceftazidime-avibactam in each disk was
$30 \mu \mathrm{g} / 20 \mu \mathrm{g}$. Testing were performed precisely according to the recommendations of the CLSI [19].

For the Etest gradient diffusion method, ceftazidimeavibactam Etest strips were obtained from BioMérieux (Marcy l'Etoile, France). The tests were performed in strict accordance with the manufacturer's instructions. The ceftazidime concentration gradient ranged from 0.016 to $256 \mu \mathrm{g} / \mathrm{mL}$ with avibactam at a constant concentration of $4 \mu \mathrm{g} / \mathrm{mL}$. When the Etest MIC value was between the standard value and twice the standard value $(0.016,0.032$, $0.064,0.125,0.25,0.5,1,2,4,8,16,32,64,128$, and 256), the high standard value was considered as the MIC.

The MH agar plates used for both the disk diffusion method and Etest gradient diffusion method for antimicrobial susceptibility testing were obtained from Oxoid.

The BMD was performed strictly following CLSI guidelines [18]. Ceftazidime and avibactam powder were obtained from MedChemExpress (Monmouth Junction, NJ, USA). The ceftazidime concentration ranged from the standard double dilution of $0.016-256 \mu \mathrm{g} / \mathrm{mL}$. The concentration of avibactam was fixed at $4 \mu \mathrm{g} / \mathrm{mL}$.

Quality controls were evaluated simultaneously in each batch of experiments. Colony counting was performed to monitor the inoculum density. Escherichia coli ATCC 25922, K. pneumoniae ATCC 700603, E. coli ATCC 35218, and P. aeruginosa ATCC 27853 were used as experimental quality control isolates. The tests were considered as valid only when the results for all quality control isolates were within the acceptable range.

The MICs and zone diameters of ceftazidimeavibactam for Enterobacterales and P. aeruginosa were interpreted according to the CLSI supplement M100 30 th edition [18]. Briefly, MICs of $\leq 8 / 4 \mu \mathrm{g} / \mathrm{mL}$ or a zone diameter of $\geq 21 \mathrm{~mm}$ indicated that the strain was susceptible, whereas MICs of $\geq 16 / 4 \mu \mathrm{g} / \mathrm{mL}$ or a zone diameter of $\leq 20 \mathrm{~mm}$ indicated that the strain was resistant.

Essential agreement (EA) indicated that the difference between the MIC value measured by Etest and the BMD did not exceed one two-fold dilution. CA indicated that interpretive category results for the Etest method or disk diffusion method were the same as those for the reference BMD using CLSI breakpoints. VME indicated that the strain was susceptible according to the Etest or the disk diffusion method but resistant according to the BMD. ME indicated that the strain was susceptible by the BMD but resistant by Etest or the disk diffusion method.

\section{Supplementary information}

Supplementary information accompanies this paper at https://doi.org/10. 1186/s12866-020-01870-z.

Additional file 1: Table S1. Organisms and distribution of carbapenem resistance mechanisms in this study. 


\section{Abbreviations}

BMD: Broth microdilution; MIC: Minimum inhibitory concentration; VME: Very major error; CLSI: Clinical \& Laboratory Standards Institute; ME: Major error; CA: Categorical agreement; EA: Essential agreement; CRKP: Carbapenemresistant Klebsiella pneumoniae; CRE: Carbapenem-resistant Enterobacterales; blakPC: Beta-lactamase gene, Klebsiella pneumoniae carbapenemase; bla $a_{\mathrm{NDM}}$ : Beta-lactamase gene, New Delhi metallo- $\beta$-lactamase; bla $a_{\mathrm{MP}}$ : Betalactamase gene, imipenemase; ATCC: American Type Culture Collection

\section{Acknowledgements}

We would like to thank BioMérieux for providing Etest strips and Editage (www.editage.cn) for English language editing.

\section{Authors' contributions}

HW conceived and designed the study. QW, FZ, ZW, HC, XW, YZ, and SL performed experiments described in this study. QW wrote the draft, and HW revised the manuscript. All authors approved the final version.

\section{Funding}

HW acknowledges support from the National Natural Science Foundation of China (NSFC), Project number: 81625014 . The NSFC did not have a role in the design of the study, in the collection, analysis, or interpretation of the data, or in writing the manuscript.

\section{Availability of data and materials}

All documents and additional data are available from the corresponding author upon reasonable request.

\section{Ethics approval and consent to participate}

Not applicable.

\section{Consent for publication}

Not applicable.

\section{Competing interests}

The authors declare that they have no competing interests.

Received: 30 April 2020 Accepted: 22 June 2020

Published online: 29 June 2020

\section{References}

1. Chinese XDRCWG, Guan X, He L, Hu B, Hu J, Huang X, Lai G, Li Y, Liu Y, Ni Y, et al. Laboratory diagnosis, clinical management and infection control of the infections caused by extensively drug-resistant gram-negative bacilli: a Chinese consensus statement. Clin Microbiol Infect. 2016;22(Suppl 1):S15-25.

2. Hu F, Zhu D, Wang F, Wang M. Current status and trends of antibacterial resistance in China. Clin Infect Dis. 2018;67(Suppl 2):S128-34.

3. Grundmann $\mathrm{H}$, Glasner C, Albiger B, Aanensen DM, Tomlinson CT, Andrasević AT, Cantón R, Carmeli Y, Friedrich AW, Giske CG, et al. Occurrence of carbapenemase-producing Klebsiella pneumoniae and Escherichia coli in the European survey of carbapenemase-producing Enterobacteriaceae (EuSCAPE): a prospective, multinational study. Lancet Infect Dis. 2017:17(2):153-63.

4. Pogue JM, Bonomo RA, Kaye KS. Ceftazidime/Avibactam, Meropenem/ Vaborbactam, or both? Clinical and formulary considerations. Clin Infect Dis. 2019;68(3):519-24

5. Shirley M. Ceftazidime-avibactam: a review in the treatment of serious gram-negative bacterial infections. Drugs. 2018;78(6):675-92.

6. Karlowsky JA, Kazmierczak KM, Bouchillon SK, de Jonge BLM, Stone GG, Sahm DF. In Vitro Activity of Ceftazidime-Avibactam against clinical isolates of Enterobacteriaceae and Pseudomonas aeruginosa collected in Latin American countries: results from the INFORM Global Surveillance Program, 2012-2015. Antimicrob Agents Chemother:2019.

7. Giddins MJ, Macesic N, Annavajhala MK, Stump S, Khan S, McConville TH, Mehta M, Gomez-Simmonds A, Uhlemann AC. Successive emergence of ceftazidime-avibactam resistance through distinct genomic adaptations in blaKPC-2-harboring Klebsiella pneumoniae sequence type 307 isolates. Antimicrob Agents Chemother. 2018;62(3):e02101-17.

8. Gaibani P, Campoli C, Lewis RE, Volpe SL, Scaltriti E, Giannella M, Pongolini $\mathrm{S}$, Berlingeri $\mathrm{A}$, Cristini $\mathrm{F}$, Bartoletti $\mathrm{M}$, et al. In vivo evolution of resistant subpopulations of KPC-producing Klebsiella pneumoniae during ceftazidime/avibactam treatment. J Antimicrob Chemother. 2018;73(6): 1525-9.

9. Tzouvelekis LS, Markogiannakis A, Psichogiou M, Tassios PT, Daikos GL. Carbapenemases in Klebsiella pneumoniae and other Enterobacteriaceae: an evolving crisis of global dimensions. Clin Microbiol Rev. 2012;25(4):682707.

10. Gutiérrez-Gutiérrez $B$, Salamanca $E$, de Cueto $M$, Hsueh P-R, Viale P, PañoPardo JR, Venditti M, Tumbarello M, Daikos G, Cantón R, et al. Effect of appropriate combination therapy on mortality of patients with bloodstream infections due to carbapenemase-producing Enterobacteriaceae (INCREMENT): a retrospective cohort study. Lancet Infect Dis. 2017;17(7): 726-34.

11. Wang $Q$, Wang $X$, Wang J, Ouyang $P$, Jin $C$, Wang $R$, Zhang $Y$, Jin $L$, Chen $H$, Wang Z, et al. Phenotypic and genotypic characterization of carbapenemresistant Enterobacteriaceae: data from a longitudinal large-scale CRE study in China (2012-2016). Clin Infect Dis. 2018;67(Suppl 2):S196-205.

12. Chen W, Sun L, Guo L, Cao B, Liu Y, Zhao L, Lu B, Li B, Chen J, Wang C. Clinical outcomes of ceftazidime-avibactam in lung transplant recipients with infections caused by extensively drug-resistant gram-negative bacilli. Ann Transl Med. 2020;8(3):39.

13. Sternbach N, Leibovici Weissman $Y$, Avni T, Yahav D. Efficacy and safety of ceftazidime/avibactam: a systematic review and meta-analysis. J Antimicrob Chemother. 2018;73(8):2021-9.

14. Shields RK, Clancy CJ, Pasculle AW, Press EG, Haidar G, Hao B, Chen L, Kreiswirth BN, Nguyen MH. Verification of ceftazidime-avibactam and ceftolozane-tazobactam susceptibility testing methods against carbapenemresistant Enterobacteriaceae and Pseudomonas aeruginosa. J Clin Microbiol. 2018;56(2).

15. Kresken $M$, Korber-Irrgang B. Performance of the Etest for susceptibility testing of Enterobacterales (Enterobacteriaceae) and Pseudomonas aeruginosa toward ceftazidime-avibactam. J Clin Microbiol. 2018;56(9).

16. Sader HS, Rhomberg PR, Chandrasekaran S, Trejo M, Fedler KA, Boyken LD, Diekema DJ. Correlation between broth microdilution and disk diffusion results when testing ceftazidime-avibactam against a challenge collection of Enterobacterales isolates: results from a multilaboratory study. J Clin Microbiol. 2020;58(4)

17. Sader HS, Rhomberg PR, Huband MD, Critchley IA, Stone GG, Flamm RK, Jones RN. Assessment of 30/20-microgram disk content versus MIC results for ceftazidime-avibactam tested against Enterobacteriaceae and Pseudomonas aeruginosa. J Clin Microbiol. 2018;56(6).

18. CLSI. Performance standards for antimicrobial susceptibility testing. 30th ed. CLSI supplement M100. Wayne: Clinical and Laboratory Standards Institute; 2020.

19. CLSI. Performance standards for antimicrobial disk susceptibility tests. 12th ed. M02-A12. Wayne, PA: Clinical and Laboratory Standards Institute, 2015.

\section{Publisher's Note}

Springer Nature remains neutral with regard to jurisdictional claims in published maps and institutional affiliations.

Ready to submit your research? Choose BMC and benefit from:

- fast, convenient online submission

- thorough peer review by experienced researchers in your field

- rapid publication on acceptance

- support for research data, including large and complex data types

- gold Open Access which fosters wider collaboration and increased citations

- maximum visibility for your research: over $100 \mathrm{M}$ website views per year

At BMC, research is always in progress.

Learn more biomedcentral.com/submissions 